\title{
A Comparison of Stimulus Presentation Methods in Temporal Discrimination Testing
}

\author{
Eavan M. McGovern \\ St Vincent's University Hospital, Dublin, Ireland \\ John Butler \\ Technological University Dublin, john.s.butler@tudublin.ie \\ Ines Beiser \\ University College Dublin, Dublin, Ireland
}

See next page for additional authors

Follow this and additional works at: https://arrow.tudublin.ie/scschmatart

Part of the Mathematics Commons, and the Medicine and Health Sciences Commons

\section{Recommended Citation \\ McGovern, E., Butler, J.S. \& Beiser, I. (2017). A Comparison of Stimulus Presentation Methods in Temporal Discrimination Testing. Physiological Measurement, vol. 38, no. 2, pg. 67-54. 10.1088/1361-6579/38/2/ N57}

This Article is brought to you for free and open access by the School of Mathematics at ARROW@TU Dublin. It has been accepted for inclusion in Articles by an authorized administrator of ARROW@TU Dublin. For more information, please contact arrow.admin@tudublin.ie, aisling.coyne@tudublin.ie,gerard.connolly@tudublin.ie.

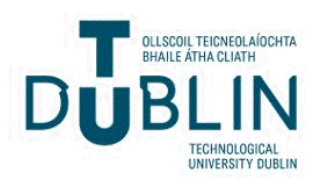




\section{Authors}

Eavan M. McGovern, John Butler, Ines Beiser, Laura Williams, Brendan Quinlivan, Shruti Narasiham, Rebecca Beck, Sean O'Riordan, Richard B. Reilly, and Michael Hutchinson 
NOTE

\section{A comparison of stimulus presentation methods in temporal discrimination testing}

To cite this article: Eavan M Mc Govern et al 2017 Physiol. Meas. 38 N57

View the article online for updates and enhancements.
Related content

Non-parametric bootstrapping method for measuring the temporal discrimination threshold for movement disorders John S Butler, Anna Molloy, Laura Williams et al.

Menstrual cycle and the temporal discrimination threshold

Eavan M Mc Govern, Emer O'Connor, Ines Beiser et al.

Estimation of temporary change of activation areas in $\mathrm{fMRI}$ measurement Tadanori Fukami, Takamasa Shimada, Takao Akatsuka et al.

\section{Recent citations}

- Abnormal somatosensory temporal
$\frac{\text { discrimination in Parkinson's disease: }}{\text { Pathophysiological correlates and role in }}$
$\frac{\text { motor control deficits }}{\text { Myung Sik Lee et al }}$




\title{
A comparison of stimulus presentation methods in temporal discrimination testing
}

\author{
Eavan M Mc Govern ${ }^{1,2,3}$, John S Butler ${ }^{3,4}$, Ines Beiser ${ }^{1,2}$, \\ Laura Williams $^{1,2}$, Brendan Quinlivan ${ }^{3}$, Shruti Narasiham ${ }^{3}$, \\ Rebecca Beck ${ }^{3}$, Sean O'Riordan ${ }^{1,2}$, Richard B Reilly ${ }^{3,5}$ \\ and Michael Hutchinson ${ }^{1,2}$
}

${ }^{1}$ Department of Neurology, St. Vincent's University Hospital, Dublin, Ireland 2 School of Medicine and Medical Sciences, University College Dublin, Dublin, Ireland

3 Trinity Centre for Bioengineering, Dublin, Ireland

${ }^{4}$ School of Mathematical Sciences, Dublin Institute of Technology, Dublin, Ireland

${ }^{5}$ School of Medicine Trinity College Dublin, The University of Dublin, Dublin, Ireland

E-mail: eavanmcgov@hotmail.com and e.mcgovern@svuh.ie

Received 13 July 2016, revised 7 October 2016

Accepted for publication 1 November 2016

Published 18 January 2017

\begin{abstract}
The temporal discrimination threshold (TDT) is the shortest time interval at which an individual detects two stimuli to be asynchronous (normal $=$ $30-50 \mathrm{~ms}$ ). It has been shown to be abnormal in patients with disorders affecting the basal ganglia including adult onset idiopathic focal dystonia (AOIFD). Up to $97 \%$ of patients have an abnormal TDT with age- and sex-related penetrance in unaffected relatives, demonstrating an autosomal dominant inheritance pattern. These findings support the use of the TDT as a pre-clinical biomarker for AOIFD. The usual stimulus presentation method involves the presentation of progressively asynchronous stimuli; when three sequential stimuli are reported asynchronous is taken as a participant's TDT. To investigate the robustness of the 'staircase' method of presentation, we introduced a method of randomised presentation order to explore any potential 'learning effect' that may be associated with this existing method. The aim of this study was to investigate differences in temporal discrimination using two methods of stimulus presentation. Thirty healthy volunteers were recruited to the study (mean age $33.73 \pm 3.4$ years). Visual and tactile TDT testing using a staircase and randomised method of presentation order was carried out in a single session. There was a strong relationship between the staircase and random method for TDT values. This observed consistency between testing methods suggests that the existing experimental approach is a robust method
\end{abstract}


of recording an individual's TDT. In addition, our newly devised randomised paradigm is a reproducible and more efficient method for data acquisition in the clinic setting. However, the two presentation methods yield different absolute TDT results and either of the two methods should be used uniformly in all participants in any one particular study.

Keywords: temporal discrimination, random, usual, Gaussian model, adult onset dystonia

(Some figures may appear in colour only in the online journal)

\section{Introduction}

The temporal discrimination threshold (TDT) is a measure of the point at which an individual determines two sensory stimuli to be asynchronous (normal 30-50 ms) (Kimmich et al 2011). It has been shown to be abnormal in neurological movement disorders affecting the basal ganglia including multiple system atrophy, Parkinson's Disease and adult onset idiopathic focal dystonia (AOIFD) (Lyoo et al 2007, Scontrini et al 2009, Bradley et al 2010, Rocchi et al 2013, Conte et al 2014, Kimmich et al 2014). Focal lesions affecting the basal ganglia may also result in abnormalities of temporal discrimination (Lacruz et al 1991), whereas lesions interrupting peripheral sensory pathways do not appear to affect temporal discrimination (Green et al 1961). Functional MRI studies (Rao et al 2001, Pastor et al 2006) indicate that the basal ganglia play an important role in temporal discrimination of sensory stimuli. Abnormal TDTs are found in non-manifesting carriers in DYT1-related dystonia and PINK1related parkinsonism (Fiorio et al 2007, 2008); as such an abnormal TDT is considered to be an endophenotype, a marker of non-manifesting gene carriage. AOIFD is an autosomal dominant condition with reduced penetrance (Waddy et al 1991, Leube et al 1997, Defazio et al 2013); endophenotypes are useful because in less than $1 \%$ of cases of AOIFD a genetic cause can be determined. Up to 97\% of patients with AOIFD have an abnormal TDT with age-related penetrance in unaffected relatives demonstrating autosomal dominant inheritance (Bradley et al 2010, Kimmich et al 2011, 2014). These findings support the use of the TDT as an endophenotype, for AOIFD (Stamelou et al 2012, Kimmich et al 2014).

Despite the expanding literature on this endophenotype, little work has been done to examine or standardise the experimental approach for examining temporal discrimination. To bridge this gap and standardise the experimental protocol, we set out to compare different methodological approaches of stimulus presentation. Our current experimental protocol (staircase stimulus presentation) uses two progressively-asynchronous stimuli (visual or tactile) presented to an individual; the TDT is taken as the first of three consecutively-reported asynchronous stimuli (Kimmich et al 2011). Both stimuli (visual and tactile) are tested four times on each side (left and right), resulting in a total of 16 trials. The time at which an individual determines two stimuli to be asynchronous can vary considerably, consequently, the experiment duration is not consistent across participants. Non-randomised, progressively asynchronous (staircase) presentation of stimuli may be perceived to contribute to a potential learning effect. However, several studies have demonstrated that TDT values do not statistically vary across repeated experimental sessions; thus suggesting that the staircase method is not affected by a learning effect (Conte et al 2016, 2010). To further explore this issue, we devised a method of randomised presentation order of stimuli that was uniform in test duration. In so doing, we aimed to develop an alternative, reproducible, efficient experimental 
paradigm to remove participant subjectivity, enhance standardisation and ease data acquisition in the clinical setting.

In a previous paper we expanded our analysis of temporal discrimination to fully characterise a single participant's data (Butler et al 2015). In this paper, we adopted this 'bootstrap' analysis and fit participant data to a cumulative Gaussian psychometric function to extract the TDT, point of subjective equality (PSE) and the just noticeable difference (JND) for each participant. The PSE corresponds to the mean value of the Gaussian and the JND is proportional to the standard deviation of this function. We previously demonstrated that the PSE represents the most sensitive measure of temporal discrimination (Butler et al 2015); thus the PSE was of particular interest in comparing different methods of stimulus presentation.

\section{Aims}

(i) To investigate any differences that exist in temporal discrimination values using two methods of stimulus presentation (staircase and randomised). (ii) To devise a reproducible, efficient, randomised approach to enhance standardisation of the experimental protocol and to facilitate data acquisition in the clinical setting.

\section{Participants and methods}

\section{Participants}

Thirty, healthy volunteers (15 women) were recruited. Exclusion criteria included current or past history of any neurological disorder. The mean age of study participants was 33.73 years (SD \pm 3.4 years). The study was approved by the Medical Research Ethics Committee at St Vincent's University Hospital. Participants were randomised as to the order of testing (staircase or randomised).

\section{Staircase TDT stimulus presentation}

Visual and tactile TDT testing using the staircase method of stimulus presentation was carried out in a single session, in a sound-proofed, darkened room. This method has been previously described (Kimmich et al 2011). Briefly, this method involves presentation of progressively asynchronous stimuli to an individual. Participants were tested for two modalities: (i) a visual task (two flashing light-emitting diodes); and (ii) a tactile task (non-painful electrical stimulation of the index and middle finger). Stimuli were presented at $5 \mathrm{~s}$ intervals with asynchronicity incrementally separated in $5 \mathrm{~ms}$ steps. Participants were instructed that each pair of stimuli will either occur at the same time, i.e. perceived as one stimulus, or they will be separated in time so that one stimulus occurs earlier than the other, i.e. perceived as two stimuli distinct from one another. For each pair of stimuli, participants responded with a one-word response, 'same' - the stimuli are perceived as one stimulus, or 'different' - the stimuli are perceived as two stimuli. The trial ended when the participant reported on three consecutive occasions that the pairs of white LEDs flashed asynchronously. The first of three asynchronous responses was taken as the TDT for that trial. This procedure was repeated four times on the left and right side of the body for both modalities (visual and tactile) resulting in a total of 16 runs per participant.

Random TDT stimulus presentation: Visual and tactile TDT testing was repeated in a randomised presentation order in the same session. Participants were once again tested for two 

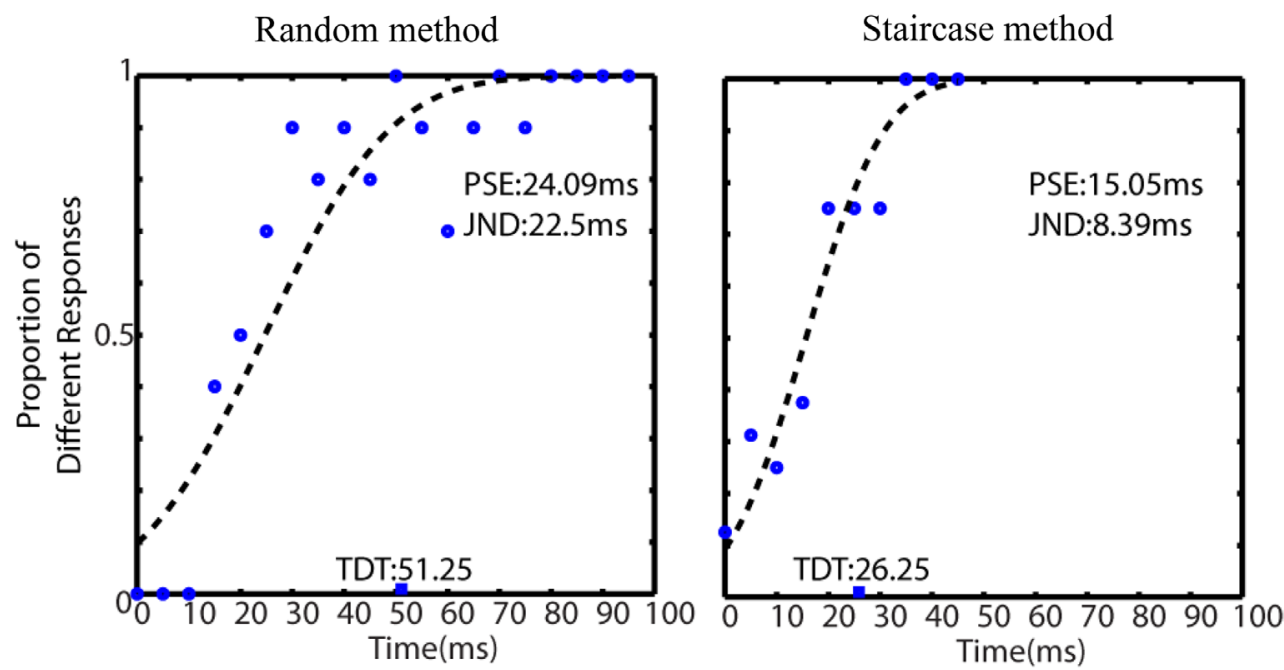

Figure 1. Data from one participant using the random and staircase method stimulus presentation during TDT acquisition. The blue circles show the proportion of perceived 'different' responses as a function of temporal asynchrony. The dashed curve represents the average cumulative Gaussian function. The blue square on the $X$-axis is the temporal discrimination threshold.

modalities. Stimuli were presented randomly, with asynchronicity varying between 0 and $100 \mathrm{~ms}$, at $5 \mathrm{~s}$ intervals. Participants were instructed to respond 'same' or 'different' following each stimulus presentation. The random TDT is calculated in the same way as the staircase approach. However, it requires one additional step; the data is first re-arranged in sequential order. The TDT for that trial is taken as the first of three asynchronous responses. The procedure was repeated four times on the left and right side of the body for both modalities (visual and tactile) resulting in a total of sixteen runs per participant.

\section{Statistical analysis}

\section{Temporal discrimination threshold}

A participant's TDT was calculated by taking the median of four runs for each modality. The results were averaged to obtain a single-combined TDT value, expressed in milliseconds (ms).

\section{Participant data psychometric fit}

A cumulative Gaussian psychometric function was fitted to the asynchronous responses. This method of TDT data analyses has been described previously (Butler et al 2015). Briefly, participant responses were coded as follows; $0=$ 'same' (synchronous), $1=$ 'different' (asynchronous). For the staircase presentation method, the number of asynchronous responses varied across trials as each trial terminated when a participant responded 'different' on three occasions. Responses were assumed to be different following termination of the run and the data was fitted to longest run across all participants. Participant asynchronous responses for the random method were also fitted to this psychometric function. The responses for both methods were averaged and plotted as a function of stimulus asynchrony and the mean and slope were extracted for each participant (figure 1). 


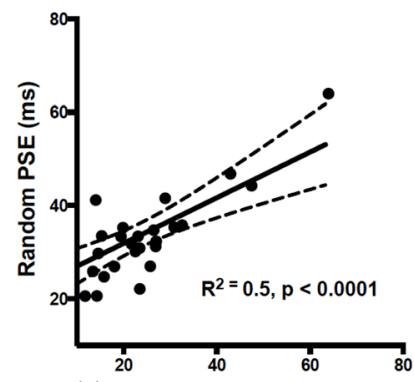

(a)

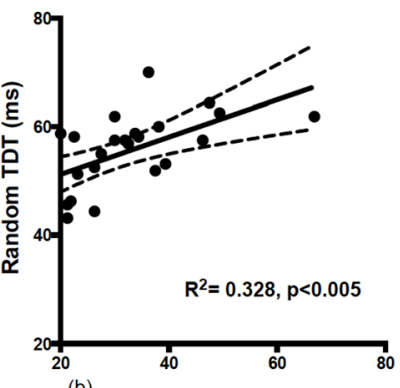

(b)

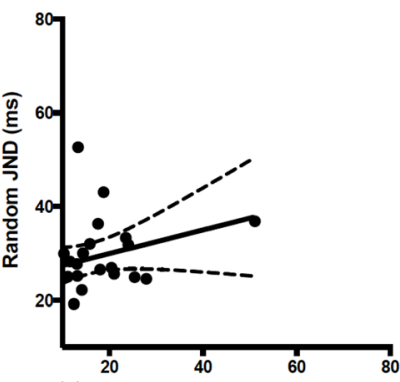

(c)

Figure 2. (a)-(c) Scatterplots of the PSE, TDT and JND values for the 30 participants determined by two different stimulus presentation techniques (staircase and random) showing the relationship between the; (a) PSE(random) and PSE(staircase), (b) TDT(random) and TDT(staircase), (c) JND(random) and JND(staircase) (PSE: perceived subjective equality. TDT: temporal discrimination threshold. JND: just noticeable difference).

The PSE is the cumulative response of 0.5 - the point at which an individual is equally likely to respond 'same' or 'different' to a stimulus. The slope of the curve represents the standard deviation of the Gaussian and is called the JND; this represents how sensitive an individual is to change around the PSE.

Comparison of staircase method and randomised method: To investigate the relationship between the two testing methods three correlation analyses were carried out:

(1) PSE(staircase) versus PSE(random); (2) TDT(staircase) versus TDT(random); (3) JND(staircase) versus JND(random). The $F$-values, mean squared error (MSE), $R$-squared values and corresponding $p$-values are reported along with $95 \%$ confidence intervals, $t$-values, and $p$-values for the intercept, and Beta value for the linear fit.

\section{Results}

Thirty participants completed the study. The mean age of study participants was 33.7 years $(\mathrm{SD} \pm 3.4$ years). Male and female participants were age-matched and equal in number. The mean values for the staircase method were: TDT $=30.57 \mathrm{~ms}$, PSE $=23.38 \mathrm{~ms}$, $\mathrm{JND}=15.95 \mathrm{~ms}$. The mean values for the random method were: TDT $55.08 \mathrm{~ms}$, PSE $33.89 \mathrm{~ms}$, JND $28.76 \mathrm{~ms}$.

To investigate the relationship between the two presentation techniques (staircase and random) the PSE, TDT and JND data were submitted to linear regression analysis. For the PSE, there was a significant relationship between the PSE(staircase) and PSE (random); PSE (random) explained a significant amount of the variance in the PSE(staircase) $\{F(1,25)=23.728$, $\left.\mathrm{MSE}=1025.773, p<0.0001, R^{2}=0.487, R_{\mathrm{ADJUSTED}}^{2}=0.466\right\}$ with a significant intercept 22.064, $\{t(26)=8.265, p<0.001 ; 95 \%$ CI $(16.566,27.562)\}$, a significant Beta $=0.490$, $\{t(26)=4.871, p<0.0001 ; 95 \%$ CI $(0.283,0.697)\}$ (figure 2(a)). For the TDT, the results also showed a significant relationship between the TDT(staircase) and TDT(random); TDT(random) explained a significant amount of the variance in the TDT(staircase) $\left\{F(1,25)=12.208, \mathrm{MSE}=463.334, p<0.005, R^{2}=0.328, R_{\text {ADJUSTED }}^{2}=0.301\right\}$ with a significant intercept 44.32, $\{t(26)=13.699, p<0.001 ; 95 \%$ CI $(37.657,50.983)\}$, a significant Beta $=0.344,\{t(26)=3.494, p<0.005$; $95 \%$ CI $(0.141,0.547)\}$ (figure $2(\mathrm{~b})$ ). For the JND, there was no significant relationship between the JND(random) and the JND(staircase) 
$\left\{F(1,25)=2.22, \mathrm{MSE}=147.7, p=0.149, R^{2}=0.082, R_{\text {ADJUSTED }}^{2}=0.045\right\}$ with a significant intercept 24.878, $\{t(26)=7.962, p<0.001 ; 95 \%$ CI $(18.443,31.3)\}$, a significant Beta $=0.252,\{t(26)=1.490, p=0.149 ; 95 \%$ CI $(-0.096,0.601)\}($ figure $2(\mathrm{c}))$.

\section{Discussion}

We have demonstrated a significant relationship between the staircase and random methods of stimulus presentation in the assessment of temporal discrimination by both the TDT and PSE measures. The strength of this observed relationship suggests that any potential 'learning effect' is consistent across participants and highlights the importance of maintaining consistency in experimental technique selection. Neurophysiological laboratories may establish normal TDT and PSE values in healthy control participants using either technique provided that the 'staircase' or 'randomised' approach is uniformly used across participants. The same technique used to generate these values should also be used when testing patients. The existing staircase method is a robust approach for assessing temporal discrimination; multi-session study designs have demonstrated consistent results across repeated experiments (Conte et al 2016, 2010). This suggests that the staircase method is unaffected by a potential learning effect. Furthermore, the temporal discrimination task requires subject familiarity with the procedure; all participants, before formal assessment of the TDT, should have an introductory trial to make them at ease with the test procedure.

The staircase method yields consistently shorter values for the TDT (by $25 \mathrm{~ms}$ ) and the PSE (by $10 \mathrm{~ms}$ ) than the random method of presentation. Some of this variability may be due to potential learning effects, not consistent between subjects. The newly devised randomised approach presents a technique potentially less susceptible to such confounders. In addition, the randomised presentation is a more efficient approach for data acquisition; an important consideration in attempting to minimise participant fatigue and facilitate increased data collection in the clinical environment. Similarly, devices with a starting procedure individualised to a participant's threshold may shorten the task to a similar extent. Irrespective of the experimental technique, our results emphasise the importance of maintaining uniformity in experimental technique selection.

Another important consideration in temporal discrimination testing is precise data analysis; a more detailed approach may enhance our understanding of this endophenotype. We have previously demonstrated that the PSE is the most sensitive measure of temporal discrimination (Butler et al 2015). Therefore, this value is particularly important when comparing different groups of participants and the effects of age and sex (Williams et al 2015). The JND represents another facet of the temporal discrimination process (Butler et al 2015) and is perhaps a less sensitive measure of the temporal discrimination process, reflected in the absence of a significant relation observed for the JND values measured by the two techniques.

Evaluation of diagnostic tests and investigations should involve careful comparison of existing methodology to refine and improve techniques currently in use (Belavý et al 2015, Saknite et al 2016, Shehab et al 2016); which in turn will enhance diagnostic yield and accuracy. This approach should also be applied when appraising preclinical biomarkers. The preclinical (endophenotype) state represents a window of opportunity for potential-disease-modifying interventions to delay or halt disease onset (Fiandaca et al 2014). Endophenotypes improve our understanding of underlying pathobiologic networks underpinning disease (Hutchinson et al 2013). Identification of dysregulated pathways can form targets for therapeutic interventions (Premi et al 2016, Rae et al 2016). Therefore, precision testing is vital when employing preclinical biomarkers (Kimmich et al 2011, Bradley et al 2012, Conte et al 2014). 


\section{Conclusion}

Our results have demonstrated a strong relationship for TDT values between the staircase and randomised stimulus-presentation methods. We have devised a novel and time-efficient randomised stimulus presentation protocol for TDT acquisition, which may facilitate data collection. Irrespective of the experimental technique, our results emphasise the importance of maintaining uniformity in experimental technique selection. This will ensure reproducible data acquisition amongst AOIFD patients and their relatives; which may in turn enhance our understanding of this condition.

\section{Acknowledgments}

This research was supported by grants from Dystonia Ireland, the Irish Institute for Clinical Neuroscience, the Foundation for Dystonia Research (Belgium) and the Trinity Centre for BioEngineering.

\section{Financial disclosures (previous 12 months)}

Eavan McGovern, John Butler, Ines Beiser, Laura Williams, Brendan Quinlivan, Shruti Narasiham, Rebecca Beck and Richard Reilly have no funding sources, financial disclosures or conflict of interests to declare.

Michael Hutchinson: serves as associate editor of the Multiple Sclerosis Journal, has received speaker's honoraria from Biogen-Idec, Bayer-Schering and Novartis and receives research grants from Dystonia Ireland, the Health Research Board of Ireland (CSA-2012-5), Foundation for Dystonia Research (Belgium) and the Irish Institute of Clinical Neuroscience. Sean O'Riordan reports receiving a speaker's honorarium from Abbvie.

\section{Ethics statement}

Written informed consent was obtained from all participants. The study was approved by the Medical Research Ethics Committee at St Vincent's University Hospital.

\section{References}

Belavý D L, Miokovic T, Armbrecht G and Felsenberg D 2015 Evaluation of neck muscle size: longterm reliability and comparison of methods Physiol. Meas. 36 503-12

Bradley D, Whelan R, Walsh R, O’Dwyer J, Reilly R, Hutchinson S, Molloy F and Hutchinson M 2010 Comparing endophenotypes in adult-onset primary torsion dystonia Mov. Disorders 25 84-90

Bradley D et al 2012 Temporal discrimination thresholds in adult-onset primary torsion dystonia: an analysis by task type and by dystonia phenotype J. Neurol. 259 77-82

Butler J S, Molloy A, Williams L, Kimmich O, Quinlivan B, O'Riordan S, Hutchinson M and Reilly R B 2015 Non-parametric bootstrapping method for measuring the temporal discrimination threshold for movement disorders J. Neural Eng. 1246026

Conte A, Leodori G, Ferrazzano G, De Bartolo M I, Manzo N, Fabbrini G and Berardelli A 2016 Somatosensory temporal discrimination threshold in Parkinson's disease parallels disease severity and duration Clin. Neurophysiol. 127 2985-9

Conte A, Modugno N, Lena F, Dispenza S, Gandolfi B, Iezzi E, Fabbrini G and Berardelli A 2010 Subthalamic nucleus stimulation and somatosensory temporal discrimination in Parkinson's disease Brain 133 2656-63 
Conte A, Rocchi L, Ferrazzano G, Leodori G, Bologna M, Li Voti P, Nardella A and Berardelli A 2014 Primary somatosensory cortical plasticity and tactile temporal discrimination in focal hand dystonia Clin. Neurophysiol. 125 537-43

Defazio G, Jankovic J, Giel J L and Papapetropoulos S 2013 Descriptive epidemiology of cervical dystonia Tremor Other Hyperkinet. Mov. 3

Fiandaca M S, Mapstone M E, Cheema A K and Federoff H J 2014 The critical need for defining preclinical biomarkers in Alzheimer's disease Alzheimer's Dementia 10 S196-212

Fiorio M et al 2007 Defective temporal processing of sensory stimuli in DYT1 mutation carriers: a new endophenotype of dystonia? Brain 130 134-42

Fiorio M et al 2008 Subclinical sensory abnormalities in unaffected PINK1 heterozygotes J. Neurol. $2551372-7$

Green J B, Reese C L, Pegues J J and Elliott F A 1961 Ability to distinguish two cutaneous stimuli separated by a brief time interval Neurology 11 1006-10

Hutchinson M et al 2013 The endophenotype and the phenotype: temporal discrimination and adultonset dystonia Mov. Disorders 28 1766-74

Kimmich O, Bradley D, Whelan R, Mulrooney N, Reilly R B, Hutchinson S, O'Riordan S and Hutchinson M 2011 Sporadic adult onset primary torsion dystonia is a genetic disorder by the temporal discrimination test Brain 134 2656-63

Kimmich O et al 2014 Temporal discrimination, a cervical dystonia endophenotype: penetrance and functional correlates Mov. Disorders 29 804-11

Lacruz F, Artieda J, Pastor M A and Obeso J A 1991 The anatomical basis of somaesthetic temporal discrimination in humans J. Neurol. Neurosurg. Psychiatry 54 1077-81

Leube B, Kessler K R, Goecke T, Auburger G and Benecke R 1997 Frequency of familial inheritance among 488 index patients with idiopathic focal dystonia and clinical variability in a large family Mov. Disorders 12 1000-6

Lyoo C H, Seung Y L, Tae J S and Myung S L 2007 Abnormal temporal discrimination threshold in patients with multiple system atrophy Mov. Disorders 22 556-9

Pastor M A, Macaluso E, Day B L and Frackowiak R S J 2006 The neural basis of temporal auditory discrimination Neuroimage 30 512-20

Premi E et al 2016 Looking for neuroimaging markers in frontotemporal lobar degeneration clinical trials: a multi-voxel pattern analysis study in granulin disease J. Alzheimers Dis. 51 249-62

Rae C L et al 2016 Atomoxetine restores the response inhibition network in Parkinson's disease Brain

Rao S M, Mayer A R and Harrington D L 2001 The evolution of brain activation during temporal processing Nat. Neurosci. 4 317-23

Rocchi L, Conte A, Nardella A, Li Voti P, Di Biasio F, Leodori G, Fabbrini G and Berardelli A 2013 Somatosensory temporal discrimination threshold may help to differentiate patients with multiple system atrophy from patients with Parkinson's disease Eur. J. Neurol. 20 714-9

Saknite I, Zavorins A, Jakovels D, Spigulis J and Kisis J 2016 Comparison of single-spot technique and RGB imaging for erythema index estimation Physiol. Meas. 37 333-46

Scontrini A, Conte A, Defazio G, Fiorio M, Fabbrini G, Suppa A, Tinazzi M and Berardelli A 2009 Somatosensory temporal discrimination in patients with primary focal dystonia J. Neurol. Neurosurg. Psychiatry 80 1315-9

Shehab H, Desouza E D, O’Meara J, Pejović-Milić A, Chettle D R, Fleming D E B and McNeill F E 2016 Feasibility of measuring arsenic and selenium in human skin using in vivo $\mathrm{X}$-ray fluorescence (XRF) - a comparison of methods Physiol. Meas. 37 145-61

Stamelou M, Edwards M J, Hallett M and Bhatia K P 2012 The non-motor syndrome of primary dystonia: clinical and pathophysiological implications Brain 135 1668-81

Waddy H M, Fletcher N A, Harding A E and Marsden C D 1991 A genetic study of idiopathic focal dystonias Ann. Neurol. 29 320-4

Williams L J, Butler J S, Molloy A, McGovern E, Beiser I, Kimmich O, Quinlivan B, O'Riordan S, Hutchinson M and Reilly R B 2015 Young women do it better: sexual dimorphism in temporal discrimination Front. Neurol. 6 1-8 